\title{
Desporto Adaptado em Portugal: do conceito à prática
}

\section{Adapted Sport in Portugal: from concept to practice}

João Paulo Saraiva ${ }^{1}$

Marisa Robina Almeida ${ }^{1}$

Carla Oliveira ${ }^{1}$

Raquel Fernandes ${ }^{1}$

Anabela Cruz-Santos ${ }^{1}$

\section{Resumo}

O Desporto Adaptado representa, por um lado, um dos mais importantes fatores promotores do sucesso educativo, inclusão e desenvolvimento psicossocial, e por outro lado, o combate ao abandono escolar e discriminação das pessoas com deficiência. $\mathrm{O}$ objetivo deste estudo centra-se no aprofundamento de algumas questões subjacentes a esta temática a nível nacional, tais como: (1) a sua origem em Portugal; (2) as modalidades mais praticadas em Portugal, quer em contexto escolar quer federado; (3) a distribuição da amostra em função da idade, do género, do local de residência e do tipo de deficiência. Foram contactadas as instituições responsáveis pela gestão da prática desportiva a nível escolar e federado, bem como pela realização e divulgação periódica dos dados censatários da população. Os resultados apontam para uma reduzida taxa de envolvimento em atividades desportivas adaptadas em Portugal, assim como uma distribuição assimétrica desta população pelo território nacional. Os resultados obtidos preconizam a realização de estudos posteriores que aprofundem as relações de causa e efeito desta realidade, contribuindo para a delineação de estratégias que as suprimam na medida do possível.

\section{Palavras-chave}

deficiência, desporto, legislação, incidência, prática

\begin{abstract}
The Adapted Sports is on the one hand, one of the most important factors that promote educational success, inclusion, and psychosocial development, and secondly, to combat school dropout and discrimination of people with disabilities. This study focuses on the deepening of some underlying issues at the national level, such as: (1) the origin in Portugal; (3) the most widely practiced sports in Portugal, either in schools or adulthood, (4) the distribution of the sample by age, gender, place of residence and type of disability. We contacted the institutions responsible for the management of sport at school level and adulthood, as well as the realization and dissemination of periodic statistic data of the population. The results point to a low rate of involvement in adapted sports activities in Portugal, as well as an asymmetric distribution of this population by the country. The results advocate the need of further studies to deepen the relations of cause and effect of this reality, contributing to devising strategies that suppress the extent possible.
\end{abstract}

\section{Keywords}

disability, sport, law, incidence, practice 


\section{INTRODUÇÃO}

O desporto, como hoje o conhecemos, tem a sua origem na história da atividade física e do movimento humano. Nos primórdios da Humanidade (3000 a.C. até 500 a.C.), o processo de seleção natural baseava-se na força e na resistência física necessárias às atividades básicas de sobrevivência, como a caça, a pesca, a defesa contra predadores e/ou território, determinando assim a lei do mais forte. Neste contexto ambiental, por vezes hostil, indivíduos com algum tipo de deficiência eram alvo de rituais de purificação, exorcismo e até sacrifício. Apenas no século $\mathrm{XX}$, devido ao elevado número de veteranos de guerra com deficiências adquiridas em combate nas grandes guerras mundiais, e a necessidade de os reabilitar, é que as sociedades ocidentais viram-se forçadas a rever as suas conceções e atitudes face à deficiência ${ }^{[1]}$. A prática desportiva passa então a ser vista e aceite como a melhor forma de intervenção, com o objetivo de promover a sua reintegração na sociedade ${ }^{[2-5]}$. Este período foi pródigo na constituição de organizações internacionais dedicadas ao fomento e à organização de competições desportivas voltadas para pessoas com deficiência, contribuindo para o surgimento e a consolidação do conceito de Desporto Adaptado. Desde então, este fenómeno tem granjeado simpatizantes e sobretudo o reconhecimento da sua importância para a inclusão destas populações numa sociedade mais justa e igualitária, assumindo-se como uma das componentes da organização social mais dinâmica ${ }^{[6]}$.

Em termos conceituais, existem imprecisões sobre a definição de deficiência, com variações relacionadas com o modelo clínico e com o modelo social, que resultam em dificuldades na aplicação e utilização de conhecimentos produzidos nesta área ${ }^{[7]}$. Nesse contexto, uma das missões da Organização Mundial de Saúde

${ }^{[8]}$ consiste na produção de classificações internacionais que representem modelos consensuais a serem incorporados pelos sistemas de saúde, visando a utilização/ uniformização de uma linguagem comum para a descrição de problemas ou intervenções ${ }^{[9]}$. Desse modo, na 54a Assembleia da OMS, realizada em Maio de 2001, foi aprovada uma nova conceção, the International Classification of Functioning, Disability and Health (ICF), cuja versão em português foi traduzida pelo Centro Colaborador da Organização Mundial da Saúde para a Família de Classificações Internacionais em Língua Portuguesa com o título de Classificação Internacional de Funcionalidade, Incapacidade e Saúde $(C I F)^{[10]}$. Segundo este documento, a deficiência é caracterizada por perda ou anormalidade da função psicológica, fisiológica ou anatómica, temporária ou permanente, incluindo a ocorrência de limitações ou a perda de um membro, órgão ou tecido, ou qualquer outra estrutura do corpo, inclusive das funções mentais. Em muitos países, a CIF tem sido utilizada na determinação de prevalência das incapacidades, aplicada à área da proteção social, saúde ocupacional, educação, concessão de benefícios, ou como forma de avaliar pacientes em reabilitação.

Em termos operacionais, Varela (1991) e Castro (1998) explicam que o Desporto Adaptado encontra-se organizado por grupos de deficiência com características etiológicas semelhantes e não por modalidades desportivas, como acontece no desporto convencional. Por conseguinte, a cada grupo de deficiência corresponde uma Federação Internacional responsável pela organização e o desenvolvimento de atividades e eventos desportivos ajustados às suas características, assim como pela sua regulamentação em colaboração com os países afiliados ${ }^{[2,5]}$.

Em Portugal, o desporto para pessoas com deficiência não se desenvolveu ao mesmo ritmo do panorama internacional, pelo que até meados da década de setenta 
a participação destas pessoas em atividades desportivas era muito esporádica ${ }^{[11]}$. Assim como o desporto internacional teve a sua origem em contextos hospitalares vocacionados para a reabilitação dos combatentes na II Guerra Mundial, em Portugal a Guerra Colonial originou um grande número de pessoas com deficiência, que ocupavam os seus tempos livres no Centro de Medicina e Reabilitação de Alcoitão ${ }^{[12]}$.

Em 1977, a Direção-Geral dos Desporto (ex- DGD), atualmente o Instituto do Desporto de Portugal (IDP), criou um setor dedicado às pessoas com deficiência. Nesse ano, é criado o Secretariado Nacional de Reabilitação (SNR). Este órgão governamental tinha como objetivo a implementação de uma política nacional de reabilitação e integração social das pessoas com deficiência, em conformidade com a Constituição da República Portuguesa. Em 1979 é formada uma comissão de trabalho com vista à elaboração dos estatutos da Federação Portuguesa de Desporto para Pessoas com Deficiência (FPDD), que viria ser oficialmente fundada apenas 9 anos mais tarde, em 1988. Para além da organização de competições desportivas à nível nacional, a FPDD tem também a seu cargo a coordenação, preparação e participação de atletas paralímpicos em eventos internacionais.

Ao nível das modalidades, a Federação Portuguesa de Desporto para Pessoas com Deficiência (FPDD) atualmente é responsável pela regulamentação e organização de 38: Andebol em Cadeira de Rodas, Atletismo, Basquetebol, Basquetebol em Cadeira de Rodas, Bilhar, Boccia, Bowling, Canoagem, Ciclismo, Equitação, Esgrima em Cadeira de Rodas, Futebol de 5, Futebol de 7, Futebol de 11, Ginástica, Goalball, Halterofilismo, Judo, Karaté, Lutas Amadoras, Natação, Orientação, Pesca Desportiva, Petra, Remo, Rugby em Cadeira de Rodas, Slalom, Surf, Taekwondo, Tênis, Tênis de Mesa, Tênis em Cadeira de Rodas, Tiro, Tiro com Arco, Vela, Voleibol, Voleibol de Praia e Xadrez.

A oportunidade da prática desportiva para pessoas com deficiência é de extrema eficácia para a promoção da qualidade de vida das mesmas ${ }^{[13]}$, assumindo-se como uma oportunidade de testar seus limites e suas potencialidades, prevenir enfermidades secundárias e promover a integração social do indivíduo ${ }^{[14]}$. Assim, através do desporto adaptado, estamos proporcionando condições para que essas populações se reconheçam como seres humanos e busquem seu desenvolvimento de forma lúdica e prazerosa, sendo-lhe reconhecidos benefícios evidentes a vários níveis, nomeadamente físicos, psicológicos e sociais, como ganhos de independência e autoconfiança para a realização de atividades diárias, além de melhorias significativas da autoestima e do autoconceito dos seus praticantes ${ }^{[15]}$.

Alguns estudos têm avaliado os índices de participação das populações sem deficiência de diferentes idades em atividades físicas e/ou desportivas ${ }^{[16-18]}$, procurando comparar os seus resultados com as orientações emanadas por instituições responsáveis pela sua quantificação e respetivo impacto para a saúde, com o intuito de aferir o êxito ou o fracasso das políticas voltadas para adoção de estilos de vida ativos adotadas em diferentes países ${ }^{[22-24]}$. Contudo, no contexto da prática desportiva desenvolvida por pessoas com deficiência, a revisão da literatura permitiu constatar a inexistência de estudos sistemáticos sobre o tema em foco no presente trabalho em países latinos, o que representa uma lacuna sobre a qual pretendemos contribuir, fornecendo dados que possam ser usados para comparação com resultados obtidos em outros países e permitam a consequente avaliação da adequabilidade das políticas voltadas para a promoção de estilos de vida ativos e com isso a manutenção da qualidade de vidas das populações com deficiência.

Assim, procuramos com o presente artigo, numa primeira fase, analisar o estado atual do desporto adaptado praticado por pessoas com deficiência em Portugal, 
nomeadamente a sua representatividade no contexto demográfico; as modalidades mais praticadas; em que zonas do país as modalidades estão mais representadas; se a faixa etária e/ou o género influenciam o nível de participação; para depois, numa segunda fase, comparar e encontrar possíveis explicações para os resultados obtidos tendo por base os (escassos) dados estatísticos de outros países.

\section{METODOLOGIA}

Para o enquadramento teórico, recorremos à $\mathrm{B}-\mathrm{On}$, base bibliográfica online que junta diversos recursos de pesquisa (PubMed, Search Direct, Web of Science, Scielo, etc). Os critérios de procura selecionados permitiram-nos consultar os trabalhos publicados nas últimas três décadas, através do cruzamento de palavras-chave especificamente relacionadas com o objetivo do trabalho (disability, sport, history, rights, rehabilitation, disabled). Relativamente ao quadro legislativo que regula o direito das pessoas com deficiência à prática de atividades físicas e desportivas, foi realizada uma pesquisa ao sítio oficial na internet do Instituto Português de Desporto e da Juventude e consultados os referidos documentos. Em relação aos dados estatísticos apresentados sobre a população portuguesa com deficiência, foi realizada uma pesquisa ao sítio oficial na internet e por contato direto (via e-mail) do Instituto Nacional de Estatística. Os dados referentes à prática desportiva em contexto escolar foram recolhidos no site da Direção-Geral de Educação (DGE). Por fim, para a recolha dos dados relativos à prática desportiva de pessoas com deficiência em Portugal, recorremos ao contato direto (via e-mail) com a Federação Portuguesa de Desporto para Pessoas com Deficiência (FPDPP), expondo-lhes os nossos objetivos e solicitando os dados pretendidos.

\section{RESULTADOS}

\section{Caracterização da população com deficiência residente em Portugal}

Tendo por base a população residente em Portugal em 2001, que segundo os Censos realizados nesse mesmo ano ascendia a 10.394.669 habitantes, observamos que as pessoas com deficiência representavam $6,1 \%$ da população, com o género masculino a superiorizar-se $(52,5 \%)$ sobre o feminino. Em relação às tipologias mais prevalentes da população portuguesa com deficiência, a Deficiência Visual foi a mais representativa (25,7\%), seguida de Deficiência Motora (25\%) e da Outra Deficiência (23\%). No caso da população feminina, a «Deficiência Visual» revelou-se como a mais incidente $(28,5 \%)$, seguida de «Outra Deficiência» e da «Deficiência Motora» (ambas com 22,4\%). Em relação à população masculina, observa-se uma situação inversa, com a «Deficiência Motora» a ser a mais prevalente (27\%), seguida da «Deficiência Visual» (23\%) e «Outra Deficiência» (24\%). Importa realçar que ambos os géneros comungam o facto de a Paralisia Cerebral ser a problemática menos representativa $(2,4 \%)$.

Apesar destes parâmetros de pesquisa não terem sido replicados nos Censos realizados em 2011, devido a substituição da variável tipo de deficiência por tipo de incapacidade, fruto de uma mudança global de paradigma assente num modelo puramente médico para um modelo biopsicossocial, consequência da adoção de um novo quadro conceptual nesta área resultante da Classificação Internacional de Funcionalidade, Incapacidade e Saúde (CIF-CJ, 2004) aprovada na 54a Assembleia Mundial de Saúde em Maio de $2001{ }^{[8,28]}$, podemos inferir o crescimento da população portuguesa com deficiência em 2011 tomando como referência o 
crescimento proporcional da população em geral (1,42\%), o que representaria um hipotético aumento aproximado de 9.000 habitantes (cerca de 645.091 indivíduos). Todavia, de acordo com dados recolhidos pelos seus Estados membros, as Nações Unidas estimam que nos países desenvolvidos, a representatividade das pessoas com deficiência ronde os $10-12 \%$ da população.

\section{Desporto Escolar para pessoas com deficiência em Portugal}

A crescente participação de jovens com deficiência em atividades desportivas desenvolvidas no âmbito do desporto escolar pode ser observada na Tabela 1. O BOCCIA destaca-se como a modalidade mais praticada por este grupo entre 2001 e 2011, seguido do GOALBALL e de DESPORTOS ADAPTADOS.

Tabela 1 - Quadro evolutivo do número de praticantes de desporto escolar para pessoas com deficiência, a nível nacional e por modalidade, nos últimos 10 anos.

\begin{tabular}{lccccccccccc}
\multicolumn{1}{c}{ Modalidades } & $01 / 02$ & $02 / 03$ & $03 / 04$ & $04 / 05$ & $05 / 06$ & $06 / 07$ & $07 / 08$ & $08 / 09$ & $09 / 10$ & $10 / 11$ & TOTAL \\
\hline BOCCIA & 63 & 135 & 114 & 239 & $*$ & $*$ & 270 & 463 & 551 & 856 & 2.691 \\
\hline GOALBALL & 15 & 55 & 13 & 10 & $*$ & $*$ & 29 & 39 & 63 & 119 & 343 \\
\hline $\begin{array}{l}\text { DESPORTOS } \\
\text { ADAPTADOS }\end{array}$ & 0 & 0 & 0 & 0 & $*$ & $*$ & 0 & 0 & 0 & 567 & 567 \\
\hline
\end{tabular}

Fonte: Direção-Geral de Educação'.

No que se refere à adesão dos alunos para a prática do BOCCIA, os anos letivos 2002/2003 e 2004/2005 registaram o maior crescimento no número de participantes (+115\%), seguido do ano letivo 2008/2009 (+72\%). Recentemente, de acordo com os dados disponíveis relativos ao ano letivo 2010/2011, o aumento observado foi o mais significativo nessa década em termos absolutos (mais 305 alunos), representando um aumento de $55 \%$ face ao número de praticantes no ano letivo anterior.

Relativamente ao GOALBALL, o ano letivo 2002/2003 registou o maior aumento no número de praticantes da modalidade $(+266 \%)$, tendo a partir de então observado uma redução acentuada nos dois ano letivos subsequentes. Contudo, a partir do ano letivo 2007/2008, foi observado novamente um aumento do número de participantes.

Apesar de, segundo os censos de 2001, as pessoas com Deficiência Visual (praticantes de GOALBALL) ilustrarem o grupo mais representativo comparativamente aos demais tipos de deficiência, observamos um baixo nível de participação dos indivíduos em idade escolar em prática desportiva (0.01\%). Este dado esteve condicionado pela existência de um número reduzido de Grupos-Equipa desta modalidade (apenas 5 a nível nacional), contrariamente ao caso do BOCCIA (46). Importa destacar o surgimento no ano letivo 2010/2011 de uma nova "modalidade", denominada «Desportos Adaptados», tendo-se verificado a participação de 567 alunos, distribuídos por 61 Grupos-Equipa a nível nacional.

\section{Desporto federado para pessoas com deficiência}

$\mathrm{Na}$ Tabela 2 podemos observar a evolução da prática desportiva por parte de pessoas com deficiência ao longo da última década, analisada segundo diferentes perspetivas.

Relativamente ao número de participantes, a época desportiva 2005/2006 foi a mais significativa, registando um aumento superior a 30\% face à anterior. A partir de então, com exceção da época 2008/2009, observou-se uma diminuição gradual no número de praticantes inscritos. No entanto, é nesta época que verificamos o 
maior envolvimento de pessoas com deficiência do género feminino em atividades desportivas (30,5\%). Por outro lado, a nível associativo, a época 2010/2011 foi a mais representativa dos últimos anos, ao qual seguiu-se um declínio até a época em curso $(-10 \%)$ no número de clubes que dispõem de atividades desportivas para pessoas com deficiência. Por fim, em termos de implantação geográfica, entre as épocas desportivas 2008/2009 e 2011/2012 a prática desportiva por parte de pessoas com deficiência estendeu-se por todo o território nacional (Portugal Continental e Regiões Autónomas).

Tabela 2 - Participação de pessoas com deficiênica em atividades desportivas federadas.

\begin{tabular}{|c|c|c|c|c|c|c|c|c|c|}
\hline ÉPOCA DESPORTIVA & $04 / 05$ & $05-06$ & $06-07$ & $07-08$ & $08-09$ & $09-10$ & $10-11$ & $11-12$ & $12-13$ \\
\hline Atletas masculinos & 1.613 & 2.134 & 2.091 & 2.018 & 1.944 & 1.839 & 1.977 & 1.954 & 1.670 \\
\hline Atletas femininos & 620 & 777 & 688 & 754 & 855 & 737 & 688 & 699 & 634 \\
\hline $\begin{array}{l}\text { Implantação geográfica } \\
\text { (N.․ de distritos) }\end{array}$ & 17 & 17 & 16 & 14 & 20 & 20 & 20 & 20 & 17 \\
\hline Clubes em atividades & 158 & 222 & 209 & 204 & 187 & 189 & 231 & 213 & 207 \\
\hline
\end{tabular}

Fonte: Federação Portuguesa de Desporto para Pessoas com Deficiência.

Ao nível da faixa etária média dos atletas com deficiência inscritos na época desportiva 2012/2013, os valores encontrados parecem ser muito similares entre homens (31,2 anos) e mulheres (30,5 anos). Através da Tabela 3, podemos observar a distribuição da amostra em função do género, do grupo etário e do tipo de deficiência dos participantes.

Tabela 3 - Distribuição da amostra em função das variáveis grupo etário, género e a tipologia da deficiência dos participantes.

\begin{tabular}{lcccccc}
\hline & ANDDI & PCAND & ANDDMOT & ANDDVIS & LPDS & N \\
\hline 0-19 anos & 53 & 6 & 6 & 1 & 6 & 72 \\
\hline 20-35 anos & 256 & 33 & 10 & 5 & 47 & 351 \\
\hline $36-50$ anos & 117 & 15 & 3 & 10 & 7 & 152 \\
\hline+51 anos & 13 & 4 & 0 & 10 & 0 & 27 \\
\hline & ANDDI & PCAND & ANDDMOT & ANDDVIS & LPDS & N \\
\hline 0-19 anos & 160 & 19 & 12 & 7 & 4 & 202 \\
\hline 20-35 anos & 660 & 105 & 84 & 34 & 78 & 961 \\
\hline 36-50 anos & 232 & 63 & 49 & 31 & 39 & 414 \\
\hline+51 anos & 32 & 13 & 24 & 23 & 12 & 104 \\
\hline
\end{tabular}

Fonte: Federação Portuguesa de Desporto para Pessoas com Deficiência.

Independentemente do tipo de deficiência apresentada, verifica-se que a maioria da amostra em estudo (57\%) situa-se na faixa etária dos «20-35 anos», observando-se uma proporção muito próxima entre homens (57\%) e mulheres (58\%) dentro de cada género. Em contraponto, a taxa mais baixa de envolvimento em prática desportiva foi encontrada na faixa etária dos «+ de 51 anos» (6\%). No entanto, tendo por base apenas o tipo de deficiência apresentada, podemos ainda observar que uma maioria significativa da amostra (86\%) é composta por pessoas com Deficiência Intelectual (67\%), Paralisia Cerebral (11\%) e Deficiência Auditiva (9\%). A partir do cruzamento das variáveis "faixa etária" e "tipo de deficiência", verificamos que a maioria dos atletas com Deficiência Auditiva (65\%), Deficiência Intelectual (60\%) e Paralisia Cerebral (53\%) pertencem à faixa etária dos «20-35 
anos». Por outro lado, na Deficiência Visual, as faixas etárias mais velhas («36-50 anos» e «+51 anos») integram uma percentagem mais elevada da amostra do género feminino ( $38 \%$ cada).

$\mathrm{Na}$ Tabela 4, podemos observar as sete modalidades mais praticadas, que representam $73 \%$ de toda a amostra em estudo, bem como a sua distribuição em função das faixas etárias estabelecidas. Tendo por base apenas a modalidade praticada, o Atletismo é a que integra o maior número de atletas inscritos (20\%), seguida da Natação (13\%) e do Futebol (12\%). Por outro lado, ao analisar a distribuição da amostra em função do grupo etário, observamos que a maioria (57\%) encontra-se na faixa etária do «20-35 anos».

Tabela 4 - Distribuição da amostra em função das variáveis idade e modalidade praticada.

\begin{tabular}{lccccc}
\hline MODALIDADES & $0-19$ anos & $20-35$ anos & $36-50$ anos & + de 51 anos & TOTAL \\
\hline ATLETISMO & 94 & 220 & 114 & 19 & 447 \\
\hline NATAÇÃO & 51 & 178 & 50 & 9 & 288 \\
\hline FUTEBOL & 26 & 205 & 48 & 4 & 283 \\
\hline FUTSAL & 42 & 140 & 46 & 6 & 234 \\
\hline BAQUETEBOL & 4 & 99 & 51 & 18 & 172 \\
\hline BOCCIA & 18 & 81 & 52 & 17 & 168 \\
\hline GOALBALL & 7 & 26 & 24 & 9 & 66 \\
\hline
\end{tabular}

Fonte: Federação Portuguesa de Desporto para Pessoas com Deficiência.

O cruzamento das variáveis "faixa etária" e "modalidades mais praticadas" permite verificar que a faixa etária dos «20-35 anos» representa mais do que metade dos praticantes de Futebol (72\%), Natação (62\%), Futsal (60\%) e Basquetebol (58\%). Considerando o nível de prática desportiva das populações mais velhas («+51 anos»), podemos observar que o Atletismo é a modalidade mais praticada (23\%), seguida do Basquetebol (22\%) e do Boccia (21\%). Por outro lado, tendo por base os hábitos desportivos das populações mais jovens («0-19 anos»), o Atletismo também é a modalidade mais escolhida (39\%), seguida da Natação (21\%) e do Futsal (17\%).

Através da Tabela 5, podemos analisar a distribuição da amostra atendendo ao grupo etário a que pertence e o distrito onde reside, sendo possível verificar que os praticantes mais jovens encontram-se no distrito de Évora e os mais velhos no distrito vizinho, em Beja, apesar da reduzida representatividade destas localidades em termos de atletas residentes.

Se dividirmos a amostra em grupos etários, constatamos que a zona norte litoral do país (Braga, Porto e Aveiro) concentra os praticantes mais jovens (72\%). Entretanto, na região centro de Portugal, o distrito de Lisboa é o mais populoso na faixa etária «20-35 anos» (15.3\%). Em relação a população adulta «36-50 anos», os dois principais distritos do país (Lisboa e Porto) concentram 49\% da amostra, com ligeira superioridade para o distrito mais a norte (28\%). Por fim, no que respeita a população mais velha (+ de 51 anos), Lisboa concentra a maior parte da população (46\%), seguido do Porto (22\%). 
Tabela 5 - Distribuição da amostra por faixa etária e por distritos.

\begin{tabular}{|c|c|c|c|c|c|c|}
\hline DISTRITO & $\begin{array}{l}\text { Média } \\
\text { (Global) }\end{array}$ & 0-19 anos & 20-35 anos & $36-50$ anos & 51 anos & $\begin{array}{l}\mathrm{N} .{ }^{\circ} \text { de Atletas } \\
\text { residentes }\end{array}$ \\
\hline Aveiro & 27,0 & 52 & 107 & 28 & 5 & 192 \\
\hline Madeira & 28,2 & 4 & 29 & 7 & 1 & 41 \\
\hline Porto & 30,9 & 68 & 376 & 158 & 28 & 630 \\
\hline Vila Real & 32,0 & 3 & 31 & 11 & 4 & 49 \\
\hline Braga & 28,5 & 77 & 256 & 93 & 10 & 436 \\
\hline Lisboa & 36,0 & 20 & 201 & 120 & 60 & 401 \\
\hline Coimbra & 31,7 & 8 & 83 & 41 & 8 & 140 \\
\hline Viseu & 31,7 & 1 & 17 & 13 & 0 & 31 \\
\hline Setubal & 28,6 & 21 & 48 & 25 & 2 & 96 \\
\hline Leiria & 31,0 & 6 & 70 & 22 & 5 & 103 \\
\hline Faro & 30,0 & 5 & 26 & 8 & 2 & 41 \\
\hline Castelo B. & 36,0 & 0 & 1 & 1 & 0 & 2 \\
\hline Açores & 33,0 & 6 & 38 & 28 & 3 & 75 \\
\hline Santarém & 35,0 & 2 & 10 & 5 & 1 & 18 \\
\hline Évora & 25,3 & 0 & 6 & 0 & 0 & 6 \\
\hline Guarda & 28,8 & 0 & 11 & 1 & 0 & 12 \\
\hline Beja & 39,7 & 0 & 0 & 3 & 0 & 3 \\
\hline
\end{tabular}

Fonte: Federação Portuguesa de Desporto para Pessoas com Deficiência.

\section{DISCUSSÃO}

O objetivo deste estudo centrou-se numa análise sobre a prática desportiva desenvolvida por pessoas com deficiência em Portugal, com especial enfoque no contexto atual, quer em termos qualitativos quer quantitativos, bem como a sua evolução ao longo dos últimos anos. A singularidade deste trabalho, quer a nível nacional como no panorama investigativo internacional, leva-nos a considerá-lo como um importante contributo para o conhecimento aprofundado do seu objeto, motivando o nosso empenho para torná-lo uma realidade e esperançosos de que a sua divulgação suscite a replicação da sua abordagem em diferentes partes do globo.

A principal dificuldade sentida na realização deste trabalho foi, sem dúvida, a escassez de estudos sobre este tema, ainda que alguns estudos internacionais encontrados na pesquisa bibliográfica abordem tópicos como os fatores motivacionais e as barreiras/obstáculos à prática desportiva na opinião de pessoas com deficiência. Esta situação constitui uma barreira ao conhecimento contextual aprofundado da realidade encontrada, na medida em que condicionou a comparação e a discussão dos resultados obtidos e consequentemente uma adequada avaliação dos recursos humanos e/ou materiais necessários à sua evolução.

Não obstante o facto deste tipo de deficiência ser o mais representativo em Portugal (27\%) e o mais envelhecido (média de 40,3 anos), começamos por observar um baixo envolvimento da população escolar com Deficiência Visual em atividades desportivas em contexto escolar, nomeadamente o Goalball. Uma explicação para esse facto poderá estar na oferta reduzida desta modalidade por parte das 
escolas, onde apenas cinco a nível nacional, num universo de 907 estabelecimentos públicos de ensino, disponibilizaram esta prática desportiva aos seus alunos. No entanto, os dados obtidos num estudo sobre os hábitos desportivos dentro e fora da escola de crianças inglesas com deficiência, alguns obstáculos à prática desportiva apontados pela amostra prendiam-se com a falta de recursos financeiros que suportassem as deslocações para os treinos, a inadequação das instalações ou até mesmo a falta de formação/preparação específica dos técnicos/professores responsáveis pelas equipas ${ }^{[29]}$.

Por outro lado, num estudo de opinião realizado com professores de Educação Física norte-americanos sobre as barreiras encontradas na inclusão de alunos com deficiência_visual nas suas aulas, verificou que, entre os principais obstáculos, estava também a falta de preparação ao nível da sua formação académica/profissional, a falta de materiais específicos na escola, a falta de programação adequada às necessidades didáticas dos alunos com esta característica e a falta de disponibilidade de horário dos professores para trabalhar apenas e especificamente com estas crianças ou jovens ${ }^{[30]}$.

No contexto federado, tomando como referência a época desportiva mais recente (2012/2013), esta pesquisa permitiu observar, numa primeira análise, o baixo envolvimento das pessoas com deficiência em atividades desportivas de caráter regular e competitivo (apenas $0,4 \%$ ) em Portugal, comparativamente ao observado em outros países onde esta realidade é substancialmente diferente, nomeadamente na Austrália, onde um estudo revelou que mais de metade da população com deficiência (53\%) praticava desporto ${ }^{[33]}$. Contudo, os dados portugueses corroboram com a realidade encontrada em outras culturas, onde Modell, Rider e Menchetti (1997) constataram que os hábitos de recreação e ocupação dos tempos livres de pessoas norte-americanas com deficiência passavam por assistir televisão e ouvir rádio ${ }^{[34]}$. Uma reduzida participação em atividades físicas foi também encontrada por Boland (2005), num estudo sobre os comportamentos de saúde e as necessidades de promoção da saúde das pessoas com deficiência na Costa Leste do EUA, que verificou que apenas $2 \%$ dos inquiridos praticavam desporto regularmente [35]. Entretanto, também nos Estados Unidos, Kaye (1997) refere que os adultos com deficiência sentem-se por vezes isolados socialmente na comunidade onde vivem e que isso acaba por afastá-los da prática desportiva ${ }^{[36]}$. Outros fatores têm sido apontados como obstáculos ao envolvimento de pessoas com deficiência em atividades físicas e/ou desportivas, desde questões estruturais, como as barreiras arquitetónicas da via pública e a falta de instalações adequadas, até questões de natureza financeira ou até mesmo emocional. Sobre as questões emocionais, Rimmer, Riley, Wang, Rauworth e Jurkowskil (2004) referem que o motivo mais citado para a relutância das pessoas com deficiência em praticar desporto foi a perceção de que estas instalações seriam ambientes hostis. Esta perceção foi muitas vezes relacionada com outras observações sobre as atitudes e os comportamentos negativos de pessoas sem deficiência, incluindo os profissionais que trabalham nessas instalações e pessoas sem deficiência que também as utilizariam. Outras barreiras emocionais foram identificadas pelos autores, nomeadamente o medo do desconhecido, a preocupação com a necessidade de solicitar a assistência de terceiros e a falta de incentivo de amigos e/ou dos familiares para participar em atividades desportivas ${ }^{[37]}$.

Em termos geográficos, verificamos que a maioria dos participantes deste estudo reside e desenvolve a sua prática desportiva nas duas principais cidades do país (Lisboa e Porto), o que pode ser explicado pela qualidade de vida que elas 
proporcionam aos seus habitantes e que está comprovada cientificamente ${ }^{[38]}$. Entretanto, analisando a distribuição da amostra por regiões, verificamos que mais de metade dos atletas (57\%) reside no norte de Portugal, enquanto o sul é a região menos representativa (6\%). Por outro lado, tendo por base uma análise centrada no cruzamento das variáveis "faixa etária" e "distrito onde reside", podemos observar que entre os três distritos mais representativos da amostra (Porto, Braga e Lisboa), Braga é o que apresenta a média de idade mais baixa (28,5 anos). Tendo por base o cruzamento das variáveis "tipo de deficiência" e "distrito de residência", podemos que observar a maioria das pessoas com Deficiência Intelectual (55\%) reside na zona norte do país, distribuída pelos distritos do Porto (30\%) e Braga (25\%), enquanto a maioria das pessoas com Paralisia Cerebral (58\%) dividem-se entre o norte e o centro, nomeadamente nos distritos do Porto (36\%) e Lisboa (22\%). No caso das pessoas com Deficiência Motora, a maioria da amostra (48\%) também divide-se pelas principais cidades do país (Porto e Lisboa), com 13\% e 35\% respetivamente. Por fim, em relação as pessoas com Deficiência Visual, a esmagadora maioria da amostra (81\%) reside na capital do país (Lisboa), enquanto a maioria das pessoas com Deficiência Auditiva (75\%) reparte-se pelas cidades do Porto (28\%) e Lisboa (48\%).

Ao nível das modalidades mais praticadas por pessoas com deficiência, observamos que o Atletismo é a mais representativa em Portugal, corroborando com os resultados obtidos por Omar-Fauzee, Mohdi-Ali, Geok e Ibrahim (2010) com atletas paralímpicos malaios ${ }^{[39]}$. Em contrapartida, entre as sete modalidades mais praticadas, o Goalball revelou-se como a menos representativa. Sobre esta situação, Ward, Famsworth, Babkes-Stellino e Perrett (2011), num estudo sobre as influências sociais que são exercidas sobre pessoas com Deficiência Visual para a prática desportiva, referem que o círculo de amigos constitui um importante fator motivacional para o seu envolvimento em atividades desportivas, enquanto os pais eram vistos apenas como fornecedores de autorização e equipamentos ${ }^{[40]}$. Entretanto, foi constatada uma participação diminuta do sexo feminino em todas as modalidades estudadas, o que, segundo este mesmos autores, destacam o gosto do sexo masculino pela prática desportiva, que a justificam pelo divertimento proporcionado e pela redução dos níveis de estresse, enquanto o sexo feminino não revelou interesse por atividades físicas em geral. Sobre esta questão, Rimmer, Rubin e Braddock (2000) constatou que menos de $10 \%$ das mulheres afro-americanas com deficiência física participavam em sessões de atividade física estruturada ${ }^{[41]}$. Do mesmo modo, um estudo realizado em 2010 pela Comissão de Desporto Australiano sobre o nível de participação das pessoas com deficiência em atividades desportivas ou recreativas revelou que as mulheres eram tendencialmente menos ativas que os homens ${ }^{[42]}$. Também entre atletas de alta competição são observados constrangimentos ao nível da participação feminina em atividades desportivas. Neste contexto, Olenik, Matthews e Steadwrad (1995), num estudo de opinião de atletas paralímpicas europeias e norte-americanas sobre as desigualdades e as barreiras no desporto adaptado feminino, referem que a participação no desporto de elite fez com que muitas mulheres com deficiência sentissem que estavam a reforçar negativamente a sua imagem, passando a ideia de um ser "diferente" ou "incapaz"[43].

Em suma, depois de apresentados os resultados do presente trabalho, e analisadas as suas características à luz da escassa literatura disponível sobre o tema em estudo, julgamos importante elencar algumas ilações que decorrem de uma reflexão crítica sobre o desporto adaptado em Portugal, nomeadamente (a) o baixo envolvimento das pessoas com deficiência, independente da sua tipologia, em atividades 
desportivas, comparativamente à outras populações, pelo que devem ser adotadas medidas de fomento e posterior manutenção de estilos de vida ativos, que vão desde da eliminação de eventuais barreiras arquitetónicas que possam condicionar/ impossibilitar a sua frequência até a formação cívica dos profissionais que nelas trabalham e dos seus familiares e amigos, sensibilizando-os para os benefícios da prática desportiva para as pessoas com deficiência assim como para a importância do seu apoio emocional; (b) o baixo envolvimento das mulheres portuguesas com deficiência, sendo necessários estudos adicionais que ajudem a clarificar o real motivo dessa omissão no contexto nacional, permitindo desta forma reduzir as assimetrias atualmente existentes; e (c) apesar de a maioria da população adulta com deficiência em Portugal que pratica desporto estar concentrada nos principais centros urbanos, e não obstante o facto de a oferta desportiva estender-se à praticamente todo o território nacional nesta faixa etária, a nível escolar ainda subsistem grandes assimetrias, que devem ser colmatadas, quer ao nível da variabilidade da oferta desportiva quer ao nível dos recursos humanos e materiais necessários à sua consecução. Para tal, consideramos de vital importância um planeamento objetivo e coerentemente estruturado tanto dos programas de formação de técnicos especializados como das escolas no que se refere aos materiais de apoio indispensáveis à prática desportiva para alunos com as mais variadas deficiências.

Os benefícios da atividade física e da participação desportiva para pessoas com deficiência também são significativos. A prática desportiva para estas pessoas impede problemas de saúde, reduzindo o risco de desenvolver doenças cardíacas, controle de peso, construção muscular magra, reduzindo a gordura e prevenção da osteoporose $^{[49]}$.

Além da prevenção de condições secundárias e promoção da saúde geral e bem -estar, a prática desportiva pode ser importante na vida do dia-a-dia das pessoas com deficiência, ao compensar o decréscimo da funcionalidade física que decorre não só do processo de envelhecimento natural mas também das mudanças relacionadas à sua condição (mobilidade reduzida, espasticidade, contraturas, dor) ${ }^{[50]}$. A força e resistência que se desenvolve através da sua participação podem ajudar a manter um alto nível de independência ${ }^{[31]}$. $\mathrm{O}$ aumento destes níveis aumenta a capacidade de um indivíduo com deficiência em ir para a escola, trabalho, e participar de uma forma mais ativa em todos os aspetos da vida da comunidade. Além destes aspetos, as pessoas com deficiência que participam em atividades desportivas podem desenvolver uma maior autoestima, melhores conceções do seu corpo e elevadas taxas de sucesso escolar; estão mais confiantes e mais propensos a concluir o ensino secundário ou até mesmo a matricular numa universidade. Ao nível das relações sociais, são também conhecidos os benefícios da prática desportiva para a inserção do indivíduo com deficiência na comunidade onde vive ${ }^{[32]}$, permitindo ainda que o desenvolvimento de competências, tais como o trabalho em equipa, a definição de metas, a busca da excelência no desempenho e outros comportamentos de realização sejam alcançados ${ }^{[51]}$.

\section{REFERÊNCIAS}

1. Guttmann L. Textbook of Sport for the Disabled. British Journal of Sports Medicine. 1976;10(4).

2. Castro JAMe. Análise de alguns aspetos na prática desportiva por pessoas deficientes. SOBAMA - Revista da Sociedade Brasileira de Atividades Motoras Adaptadas. 1998;3(3):31-4.

3. Guttmann L. Antologia desportiva: o desporto para deficientes. Lisboa: Ministério da Educação e Investigação Científica 1977. 
4. Varela A. O desporto para pessoas com deficiência. Expressão distinta do desporto. Revista Educação Física Especial. 1991;5-6:53-62.

5. Bento JO. Desporto, Saúde, Vida. Em defesa do Desporto. Lisboa: Livros Horizonte 1991.

6. Amiralian MLT, Pinto EB, Ghirardi MIG, Lichtig I, Masini EFS, Pasqualin L. Conceituando deficiência. Rev Saúde Pública. 2000;34(1):97-103.

7. OMS. Classificação Internacional de Funcionalidade, Incapacidade e Saúde. Lisboa: DireçãoGeral de Saúde 2004:238.

8. Farias N, Buchalla CM. A Classificação Internacional de Funcionalidade, Incapacidade e Saúde da Organização Mundial da Saúde: Conceitos, Usos e Perspectivas. Rev Bras Epidemiol. 2005;8(2):187-93.

9. Classificação Internacional de Funcionalidade, Incapacidade e Saúde. In: Direção Geral de Saúde, ed. Lisboa: Ministério da Saúde, 2004.

10. Freire MF. A inclusão através do desporto adaptado: o caso português do basquetebol em cadeira de rodas. Coimbra: Universidade de Coimbra; 2010.

11. Carvalho J. Breve História das Organizações Desportivas: Federação Portuguesa de Desporto para Pessoas com Deficiência 1995.

12. Yazicioglu K, Yavuz F, Goktepe AS, Tan AK. Influence of Adapted Sports on quality of life and life satisfaction in sport participants and non-sport participants with physical disabilities. Disabil Health J. 2012;5(4):249-53.

13. Melo ACR, López RFA. O Esporte Adaptado. Revista Digital. Buenos Aires 2002.

14. Blauwet C, Willick SE. The Paralympic Movement: Using Sports to promote health, disability rights and social integration for athletes with disabilities. Physical Medicine and Rehabilitation. 2012;4(11):851-6.

15. Caspersen CJ, Pereira MA, Curran KM. Changes in physical activity patterns in the United States, by sex and cross-sectional age. Medicine \& Science in Sports \& Exercise. 2000:1601-09.

16. Riddoch CJ, Andersen LB, Wedderlopp N, Harro M, Klasson-Heggebo L, Sardinha LB, et al. Physical Activity Levels and Patterns of 9-and 15-yr-Old European Children. Medicine \& Science in Sports \& Exercise. 2004:86-92.

17. Trost SG, Pate RR, Sallis JF, Freedson PS, Taylor WC, Dowda M, et al. Age and gender differences in objectively measured physical activity in youth. Medicine \& Science in Sports \& Exercise. 2002:350-55.

18. Global recommendations on physical activity for health. Switzerland: World Health Organization; 2010.

19. Tremblay MS, Warburton DER, Janssen I, Paterson DH, Latimer AE, Rhodes RE, et al. New Canadian Physical Activity Guidelines. Appl Physiol Nutr Metab. 2011;36:36-46.

20. Warburton DER, Katzmarzyk PT, Rhodes RE, Shephard RJ. Evidence-informed physical activity guidelines for Canadian adults. Appl Physiol Nutr Metab. 2007;32:S16-S68.

21. Mont D. Measuring Disability Prevalence. Social Protection Discussion Papers. Washington, DC 2007.

22. Disability Survey 2000: Survey of young people with a disability and sport. Leicestershire. Eng.: Sport England; 2000.

23. Lieberman LJ, Houston-Wilson C, Kozub FM. Perceived Barriers to Including Students with Visual Impairments in General Physical Education. Adapted Physical Activity Quarterly. 2002;19:364-77.

24. Participation in Sport by People with a Disability. Camberra. AUS: Australian Bureau of Statistics; 2009 04/12/2009.

25. Modell SJ, Rider RA, Menchetti BM. An exploration of the influence of educational placement on the community recreation and leisure patterns of children with developmental disabilities. Perceptual and Motor Skills. 1997;85 (2):695-704.

26. Boland M. Health promotion and health promotion needs assessment of people attending disability services in the HSE, East Coast Area. Dublin. Ireland: University College Dublin; 2005.

27. Kaye S. The status of people with disabilities in the United States. Disability Watch. Volcano, CA: Volcano Press, Inc. 1997.

28. Rimmer JH, Riley B, Wang E, Rauworth A,Jurkowski J.Physical Activity Participation Among Persons with Disabilities: Barriers and Facilitators. Am J Prev Med. 2004;26(5):419-25. 
29. Manso JRP, Simões NM. Os Municípios e a qualidade de vida em Portugal: proposta metodológica com vista à sua mensuração e ordenação. Covilhã: Universidade da Beira Interior; 2007.

30. Omar-Fauzee MS, Mohd-ali M, Geok SK, Ibrahim N. The participation motive in the Paralympics. Journal of Alternative Perspetives in the Social Sciences. 2010;2(1):250-72.

31. Ward S, Farnsworth C, Babkes-Stellino M, Perrett J. Parental influence and attraction to physical activity for youths who are visually impaired at a residential-day school. Journal of Visual Impairments. 2011;54(3):493-8.

32. Rimmer JH, Rubin SS, Braddock D. Barriers to Exercise in African AmericanWomenWith Physical Disabilities. Arch Phys Med Rehabil. 2000;81:182-88.

33. Participation and non-participation of people with disability in sport and active recreation. Sidney: Australian Sports Commission research project, in collaboration with the University of Technology; 2010.

34. Olenik LM, Matthews JM, Steadward RD. Women, Disability and Sport. Unheard Voices. Canadian Woman Studies/Les Cahiers de la Femme. 1995;15(4):54-7.

35. UnitedNations. The Standard Rules on the Equalization of Opportunities for Persons with Disabilities (20 December 1993) A/Res/48/96 online: http://www.un.org/esa/socdev/enable/ dissre00.htm. 1993 [cited; Available from:

36. Figueiredo MHA. Estudo Comparativo do Autoconceito em Alunos com Necessidades Educativas Especiais. Porto: Dissertação de Mestrado. Faculdade das Ciências do Desporto e da Educação Física - Universidade do Porto. ; 2000.

37. Boslaugh SE, Andresen EM. Correlates of physical activity for adults with disability. Centers for Disease Control and Prevention. 2006;3(NO. 3):A78.

Endereço para Correspondência
João Paulo Saraiva Santos
Rua Francisco Mendes, $n .^{\circ}$ 34-A R/C
- DRT
$4715-243$ Lamaçães
Braga - Portugal
Telemóvel: 00351968620070

Recebido 20/07/2013

Revisado 19/09/2013

$21 / 11 / 2013$

Aprovado 09/12/2013

1 À Direção-Geral de Educação foram solicitados esclarecimentos sobre a ausência de dados relativos aos anos letivos 2005/2006 e 2006/2007, assim como sobre que modalidades desportivas integram a categoria "Desportos Adaptados", não tendo obtido qualquer reposta até a edição final do presente trabalho. 